\title{
A New Medical Image Segmentation Technique Based on Variational Level Set Method
}

\author{
Mohammad N. Nobi, Faruk Chowdhury, Faysal Mahmood, and Mohammad A. Yousuf
}

\begin{abstract}
The main objective of medical image segmentation is to extract and characterize anatomical structures with respect to some input features or expert knowledge. This paper describes a way of medical image segmentation based on level set method to extract the region of interest of various medical images such as magnetic resonance imaging (MRI), computer tomography (CT), and positron emission tomography (PET) image. The overall technique of the paper is divided into three steps. The first step is to do threshold of the input image to make the entire pixel under threshold value to 0 and others to take the value as original image. This helps to keep original properties of original image and keep all value fixed as original image except the pixel that are not necessary for our analysis. Next, a morphological technique is used to remove some small ignorable parts. Finally we apply variational level set method for final segmentation. The experimental results show the efficacy of the proposed method.
\end{abstract}

Index Terms-Active contour, Medical image segmentation, thresholdng, Variational level set method.

\section{INTRODUCTION}

One of the most important problems in image processing and analysis is segmentation [1]-[3]. Segmentation is defined as partitioning portions of an image. Image segmentation plays a crucial role in many medical imaging applications by automating or facilitating the delineation of anatomical structures and other regions of interest. Medical image segmentation is a challenging task due to the various characteristics of the images, which leads to the complexity of segmentation. In the domain of biomedical image processing, correct image segmentation would aid physicians greatly in providing visual means of inspection of automatic structures. Because of some properties of medical images, such as low image contrast, noise, and diffuse boundary, medical image segmentation often face some difficult challenges.

Image segmentation is a traditional issue in image processing. There have been many researches on this topic, and many methods have been proposed such as zero crossing, thresholding, region based segmentation, watershed and level set method. Some of them are gradientbased and are vulnerable to week edges. Some of methods are intensity-based are vulnerable to noises. But the medical images have both of these two properties that we need to

Manuscript received May 9, 2011; revised July 7, 2011.

The authors are with the Department of Computer Science and Engineering, Mawlana Bhashani Science and Technology University, Tangail, Bangladesh. (e-mail: m_nurnobi@yahoo.com, faruk_ce06003@ yahoo.com, faruk.mbstu@gmail.com, faysal_mbstu06@yahoo.com, usuf672@yahoo.com ) conquer. The bulk of the earlier segmentation methods are roughly categorized based on the image features used for segmentation, into two basic approaches, the edge-based and region-based methods [4].

In this paper, we use a new mechanism based on the variational level set method incorporated with some mechanism to do medical image segmentation because of its some advantages. Our proposed technique works better for various images, not only for specific image. This makes the new technique more convenient than others. We use the thresholding such a way that doesn't make the image binary, rather it provides another image in which entire pixels less than threshold value is 0 and other keeps its original value. Then we apply morphological erosion to remove unnecessary parts of image. The erosion process in our proposed method is not always used. After applying thresholding technique, if the image contains some small ignorable parts, the erosion process is used remove the unnecessary parts from the image. Finally the variational level set method is used to do final segmentation. This variational process have some advantages, such as a significantly larger time step can be used to for numerically solving the evaluation partial differential equation, and therefore speed up the curve evaluation. Second, the level set function can be initialized with general functions that are more efficient to construct and easier to use in practice.

In the following sections, we give necessary background, describe our method and its implementation, and provide experimental results that show the overall characteristics and performance of this method. In this paper, a new algorithm based on a variational level set method is proposed. The work is organized as follows. In section 2, Level set method is described. In section 3, the proposed method is described. In section 4, some experimental results are presented that show the overall characteristics and performance of this method. Finally, the paper is concluded in section 5 .

\section{LEVEL SET MethodS}

The original level set method [5] was first proposed as a numerical technique that tracks an evolving contour. The evolving contour deforms with a speed $\mathrm{F}$ that is based on the contour curvature and image features like gradient. The curvature component in the speed keeps the contour propagating smoothly. For a given image $\mathrm{u}_{0}$, a level set function $\varphi$ is used to describe the desired contour. The $\varphi$ function has the same size with image $\mathrm{u}_{0}$, which means each pixel of image $\mathrm{u}_{0}$ will have a corresponding $\varphi$ value. We define the region where $\varphi=0$ as the contour $\mathrm{C}$, and the $\varphi>0$ region as inside the contour and the $\varphi<0$ region as outside the contour, as shown in Fig. 1. 


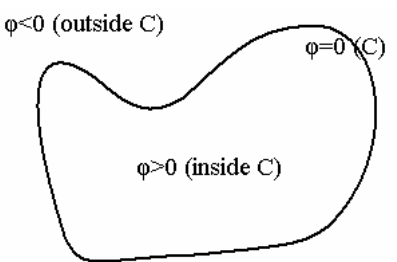

Fig. 1. Use level set function to represent contour

We can easily know that when the $\varphi$ value changes, the corresponding $\varphi>0$ region and $\varphi<0$ region will also change, therefore the boundary of $\varphi>0$ region and $\varphi<0$ region, i.e. $\varphi=0$, will be different and thus the contours evolves. After the original level set method [5] was proposed, which had no energy minimization, some researchers applied the level set formulation with a contour energy minimization in order to obtain a good convergence. This modification produced a relaxed initial condition requirement and the ability to handle the contour topology changes naturally. The modification resulted in various geodesic deformable models [6]-[16].

\section{PROPOSED METHOD}

First we will apply thresholding on the medical image remove background. Then we will apply morphological erosion to remove unnecessary part of image. Finally variational level set formulation without re-initialization has been applied for final segmentation.

\section{A. Thresholding}

Thresholding is the first steps to segment the image more precisely. Normally the thresholding method is used to make an image binary. But this technique here is used in new moderated way. Instead of making an image binary, a threshold value is defined to make all the value under threshold to 0 value and others to take the value as original image. This helps to keep original properties of original image and keep all value fixed as original image. Thus further analysis will be based on original image. The main purpose of using this technique is to ignore unnecessary part of image that is not requiring for image segmentation.

$$
g(x, y)= \begin{cases}f(x, y) & \text { if } f(x, y)>T \\ 0 & \text { if } f(x, y) \leq T\end{cases}
$$

Equation 1 states that, a threshold value $T$ is chosen. The value under $T$ is 0 and others are as like as original image $f(x, y)$. As a result it gives easily the required part. Figure 2 demonstrates this process more precisely. In Fig. 2(a), MRI image of human brain is shown and Fig. 2(b) shows the final output image after thresholding. Here the threshold value is considered 201 .

\section{B. Application of morphological erosion}

In binary morphology, an image is viewed as a subset of a Euclidean space or the integer grid, for some dimension $d$. The basic idea in binary morphology is to probe an image with a simple, pre-defined shape, drawing conclusions on how this shape fits or misses the shapes in the image. This simple "probe" is called structuring element, and is itself a binary image.

In the proposed segmentation method, the erosion process is used to remove unused or small part of image. We have stated before that the erosion process in our proposed method is not always used. This decision is taken according to image characteristics, how much the image is complex or how many small fragments are in the image after applying the thresholding technique. Basically the erosion process is used to shrink an image. After applying the thresholding technique, the image may contain some small ignorable parts that are needed to be ignored in practice. For this purpose, a morphological technique is used known as erosion. But, in case of erosion a very small sized structured element is considered so that the original part of image is not removed. In Fig. 3 (a), it is shown that the image after applying thresholding process contains several small fragments all over the image which are needed to be ignored. Erosion technique is used to remove these small fragments. Fig. 3(b) shows that most of them are removed after applying erosion technique is used.

In our proposed method, thresholding is used to determine the edges of the image. These edges will be further used for variational level set method. But, for complex images and the images that contains different colored pixel in a small area of object are not always give specific edge representation, instead it gives an image that contains several small fragments. In this situation we use erosion technique in our proposed technique for accurate edge representation. Then this image will finally used as the input image in edge indicator function in variational level set method.

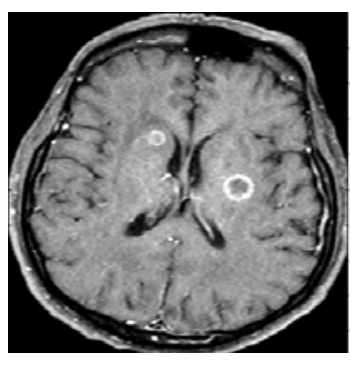

(a)

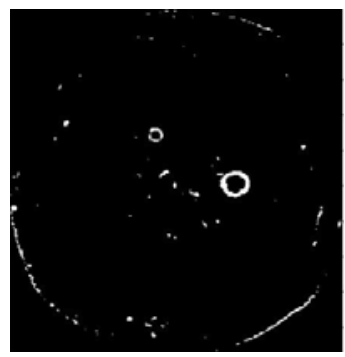

(b)
Fig. 2. (a) Input MR image of human brain (b) MR image after threshold

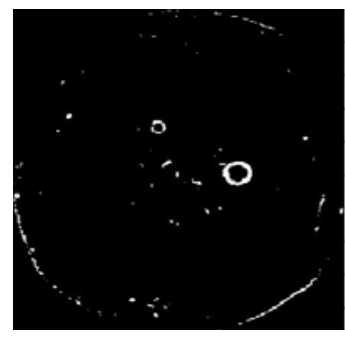

(a)

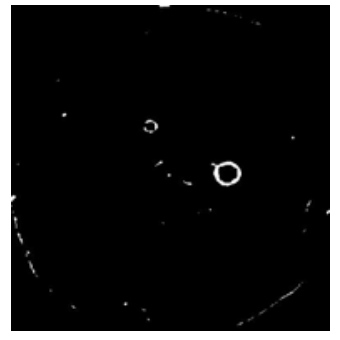

(b)
Fig. 3. (a) Threshold MR image (b) MR image after erosion

\section{Implementation of variational level set method}

A new variational level set method is used to complete segmentation process. Instead of using traditional level set method, variational level set method is used to get better result. As traditional level set method re-initialize its sign distance function in each contour evaluation, the variational level set method update its contour automatically without re-initializing.

The variational technique is implemented in some steps. First the image is smoothed using Gaussian Convolution. 
Then we apply edge indicator function to the image after erosion or after thresholding defending on the quality of the image. To achieve this goal, we explicitly define an external energy that can move the zero level curves toward the object boundaries. Let I be an image, and $g$ be the edge indicator function defined by

$$
\mathrm{g}=\frac{1}{1+\left|\nabla \mathrm{G}_{\sigma} * \mathrm{I}\right|^{2}}
$$

where $G_{\sigma}$ is the Gaussian kernel with standard deviation $\sigma$.

Then find coefficient of the internal (penalizing) energy term that helps contour to outside the object boundary. We define an external energy for a function $\emptyset(x, y)$ as below

$$
\varepsilon_{\mathrm{g}, \lambda, \mathrm{v}}(\varnothing)=\lambda \mathcal{L}_{\mathrm{g}}(\varnothing)+\mathrm{vA}(\varnothing)
$$

where $\lambda>0$ and $v$ is constant, and the terms $\mathcal{L}_{g}(\varnothing)$ and $A_{g}(\varnothing)$ are defined by

$$
\mathcal{L}_{\mathrm{g}}(\varnothing)=\int_{\Omega} \mathrm{g} \delta(\varnothing)|\nabla \emptyset| \mathrm{dxdy}
$$

and

$$
A_{g}(\varnothing)=\int_{\Omega} g H(-\varnothing) d x d y
$$

where $\delta$ is the univariate Dirac function, and $H$ is the Heaviside function. Now, we define the following total energy functional

$$
\varepsilon(\varnothing)=\mu \mathcal{P}(\varnothing)+\varepsilon_{\mathrm{g}, \lambda, v}(\varnothing)
$$

The external energy $\varepsilon_{\mathrm{g}, \lambda, v}$ drives the zero level set toward the object boundaries, while the internal energy $\mu \mathcal{P}(\varnothing)$ penalizes the deviation of $\varnothing$ from a signed distance function during its evolution. Fig. 4 shows the output image after applying variational level set method. Fig. 5 illustrates the flow chart of the proposed image segmentation method.

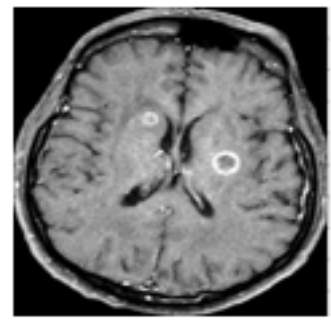

(a)

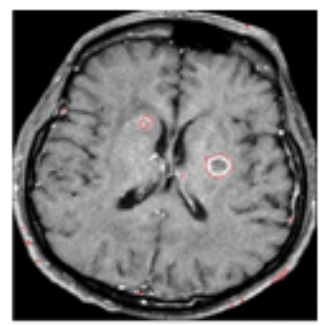

(b)
Fig. 4. (a) Input MR image (b) MR image after applying proposed method

\section{Contour evaluation}

This is very crucial to in implicit active contours is the placement of the initial contour. Since the contour moves either inward or outward, its initial placement will determine the segmentation that is obtained. For example, if there is a single object in an image, an initial contour placed outside the object and propagated inward will segment the outer boundary of the object. However, if the object has a hole in the middle, it will not be possible to obtain the boundary of this hole unless the initial contour is placed inside the hole and propagated outward. It should be noted that more than one closed curve can be used for initialization of the zero-the level set. Fig. 6 shows the whole iteration process for segmentation on MRI human brain image. First image shows the image after applying initial level set. Second image is after 200 iterations, next image after applying 360 iterations, then the image shown after iterate 480 times, next image is for image after applying 580 times. Last image shows that image after completing all the iterations.

\section{EXPERIMENTAL RESULT}

In this section, some experimental results of the proposed medical image segmentation method are presented. The proposed image segmentation technique based on variational level set method has been applied to a variety of real images in different modalities. Our proposed method works well for other types of image such as CT image of human brain and PET image of human body.

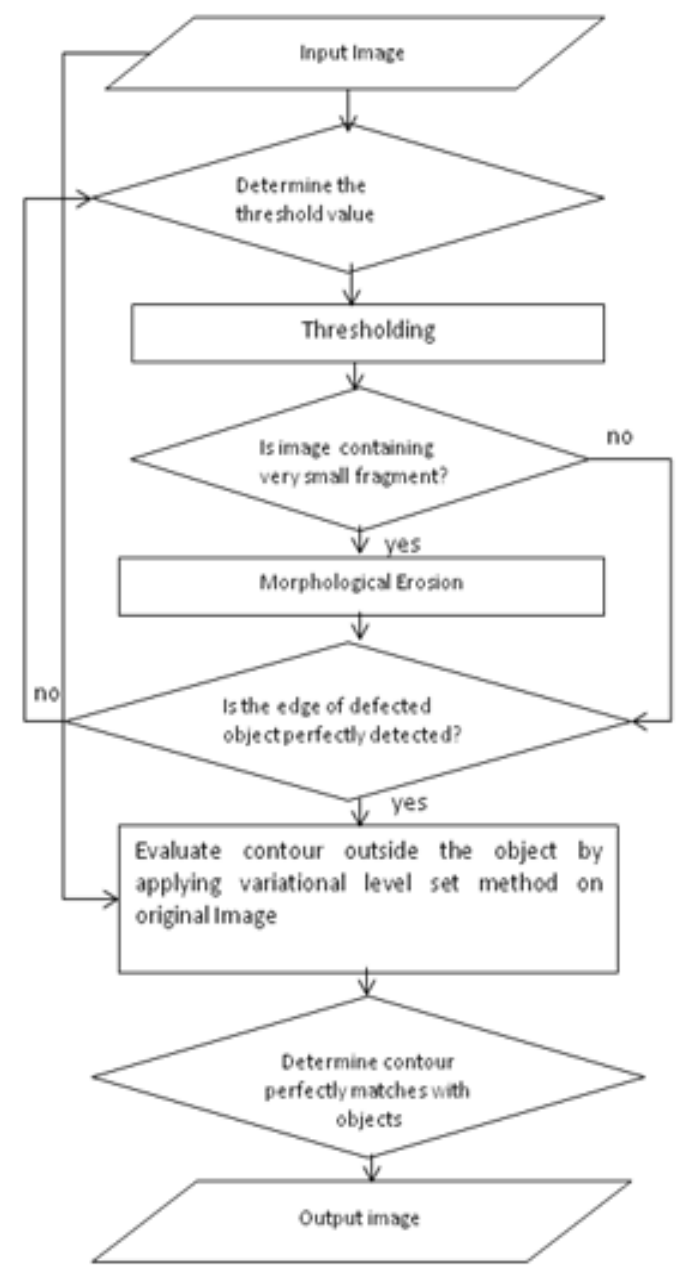

Fig. 5. Flow chart of the proposed image segmentation method

The image size for PET image that we have considered is 272x307. For PET image, we use threshold value 45. After applying thresholding technique, we got a clear image that has no unexpected part. As a result we didn't use any erosion technique for this image. We directly applied level set technique on the original image. After 620 iterations we got our final image. Although the image is too large, it didn't take lots of time for evaluation. Fig. 7 shows the PET image and the output image after applying proposed image segmentation method. CT image that we have considered is smaller than the PET image. We use the CT image of human brain. The size of the CT image that we have considered is $256 \times 256$. For CT image, we have used two threshold values 180 and 220 . The pixels that are less than 180 and greater than 220 is changed to 0 and rest pixels are kept as original image. After applying thresholding technique, we get image with some small unexpected parts. As a result, we need to use erosion technique to remove these small fragments. Then we applied variational level set technique on the original image. After 550 iterations we got 
our final image. Fig. 8 shows the CT image and the output image after applying proposed image segmentation method.

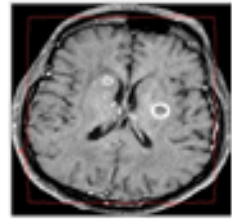

60 iteration

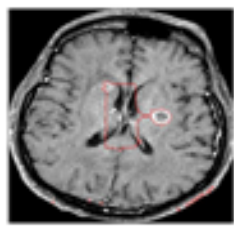

$4 \$ 0$ iteration

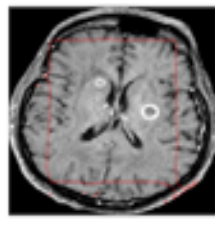

200 iteration

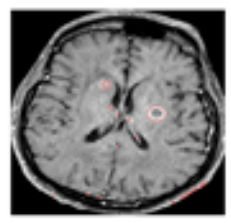

Sso iteration

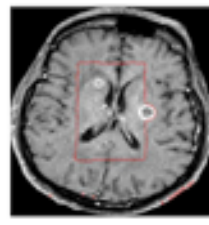

360 iteration

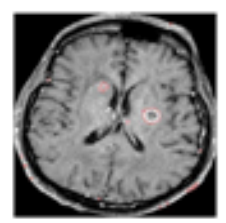

Iteration complete
Fig. 6. Contour evaluation

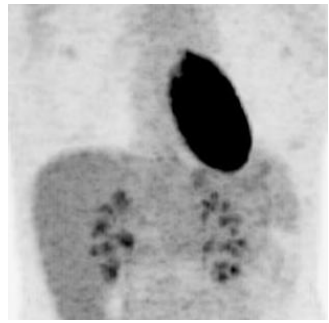

(a)

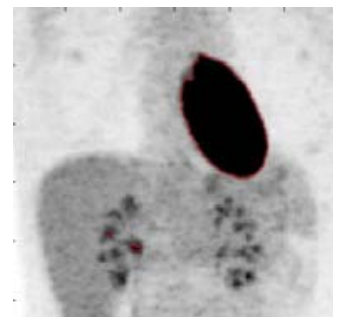

(b)
Fig. 7. (a) Input PET image (b) PET image after applying proposed method

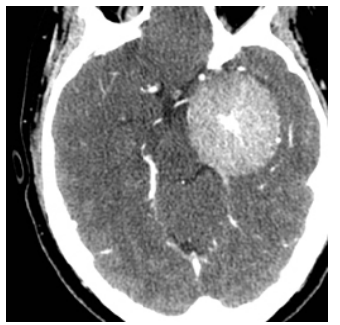

(a)

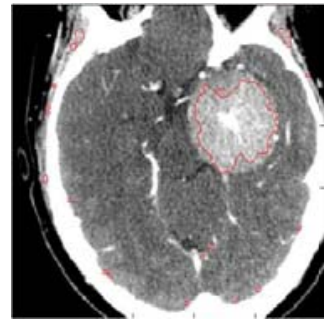

(b)
Fig. 8. (a) Input $\mathrm{CT}$ image (b) $\mathrm{CT}$ image after applying proposed method

\section{DISCUSSIONS AND CONCLUSION}

The main purpose of this paper was to segment specific object from different medical images like as MRI, CT , PET etc. In this paper, we propose a new image segmentation technique based on variational level set method and some sort of existing technique specially thresholding. The variational level set method is one of latest approach that is widely used in image processing. This variational process have some advantages, such as a significantly larger time step can be used for numerically solving the evaluation partial differential equation, and therefore speed up the curve evaluation [17]. Second, the level set function can be initialized with general functions that are more efficient to construct and easier to use in practice. Despite this method is more convenient to segment image and provide excellent for single object, but in case of detection of specific objects this method suffers for several shortcomings. First of all, this technique failed to detect specific object for more complex medical images. It works by considering whole image as single object as images has its own boundary.

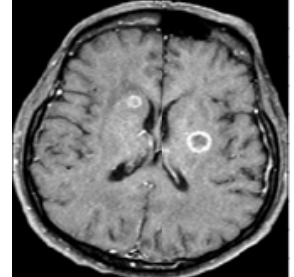

(a)

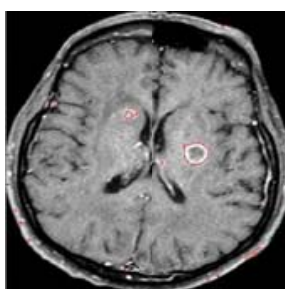

(b)
Fig. 9. (a) Input MRI human brain image (b) MRI image after applying proposed method

To make a comparison between our proposed method and the variational level set method without re-initialization, we use same MRI image of human brain that we already used for our proposed method. This image almost complex than others. This image contains two defected parts inside the image. But variational level set method is failed to segment these two objects. The contour can't enter into the image boundary. Our proposed method solves this problem by modifying the technique in more convenient way. Fig. 9 shows the result of our proposed method and Fig. 10 illustrates the variational level set method without reinitialization. From Fig. 10 it is shown that for complex medical image the variational level set method without reinitialization is failed to segment the desire object. In our experiment we shown that the situation after applying variational level set method on original MR image for 400 iterations is quite same as the image after applying 800 iterations that is shown in Fig. 10(a and b). It proves that this is not possible to enter the contour into the image to detect specific object.

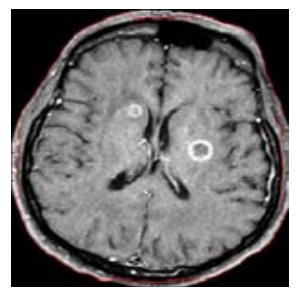

(a)

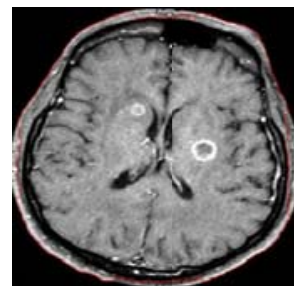

(b)
Fig.10. MRI human brain image after applying variational level set method without re-initialization (a) after 400 iterations (b) after 800 iterations

Although our proposed method provides some remarkable opportunities to segment specific object from these images but it has some limitations, such as it depends on defected object's intensity and high complexity in case of very large image.

\section{REFERENCES}

[1] L. J. van Vliet., I. T. Young, and G. L. Beckers, "An edge detection model based on non - linear laplace filtering", Pattern Recognition and Artifical Intelligence, 1988, pp. 63-73.

[2] L.J. van Vliet, I. T. Young, and G. L. Beckers "A nonlinear Laplace operator as Edge Detector in Noisy Images", Computer Vision, Graphics, and Image Processing, 1989, 45(2), pp.167-195.

[3] T. Asano, and N. Yokoya, "Image segmentation schema for low-level computer vision”, Pattern Recognition, 1981, 14, pp. 267-273.

[4] A. Chakraborty, J.S. Duncan, "Game-theoretic integration for image segmentation”, IEEE Trans. Pattern Anal. Machine Intell., 1999, 21(1), pp.12-30.

[5] R. Malladi, J. Sethian, B. Vemuri, "Shape modeling with front propagation”, IEEE Trans. Pattern Anal. Machine Intell., 1995, 17(2), pp.158-171.

[6] V. Caselles, R. Kimmel, G. Sapiro, Geodesic active contours, Int. J. Comput. Vis., 1997, 22(1), pp.61-79. 
[7] X. Wang, L. He, W.G. Wee, "Deformable contour method: a constrained optimization approach", Int. J. Comput. Vis., 2004,59(1),pp.87-108.

[8] K. Siddiqi, Y.B. Lauzie're, A. Tannenbaum, S.W. Zucker, “Area and length minimizing flows for shape segmentation", IEEE Trans. Image Process., 1998,7(3),pp.433-443.

[9] M. Rousson, N. Paragios, "Shape priors for level set representations", in: Proceedings of the Seventh European Conference on Computer Vision (ECCV), 2002, pp.78-92.

[10] T.F. Chan, L.A. Vese, "Active contour without edges", IEEE Trans. Image Process., 2001, 10, pp.266-277.

[11] N. Paragios, R. Deriche, "Geodesic active region and level set methods for supervised texture segmentation", Int. J. Comput. Vis.,2002,46(3), pp. 223-247.

[12] A. Yezzi, S. Kichenassamy, A. Kumar, P. Olver, A. Tannenbaum, "Geometric snake model for segmentation of medical imagery", IEEE Trans. Med. Imaging, 1997, 16(2), pp.199-209.
[13] W.J. Niessen, B. Romeny, M.A. Viergever, "Geodesic deformable models for medical image analysis", IEEE Trans. Med. Imaging, 1998, 17(4), pp.634-641.

[14] L.D. Cohen, R. Kimmel, "Global minimum for active contour models: a minimal path approach", Int. J. Comput. Vis., 1997, 24(1),pp.57-78.

[15] Y. Chen, S. Thiruvenkadam, F. Huang, H.D. Tagare, D. Wilson, E.A. Geiser, "On the incorporation of shape priors into geometric active contours", in: Proceedings of the IEEE Workshop on Variational and Level Set Methods, 2001, pp.145-152.

[16] M. Leventon, E. Grimson, O. Faugeras, "Statistical shape influence in geodesic active contour", Proc. IEEE Comput. Vis. Pattern Recognit.(CVPR), 2000, pp.316-322.

[17] C. Li, C. Xu, C. Gui, and M. D. Fox, "Level Set Evolution Without Re-initialization: A New Variational Formulation", IEEE International Conference on Computer Vision and Pattern Recognition (CVPR), San Diego, 2005, 1, pp. 430-436. 